\title{
Cardiac Radiosurgery for the Treatment of Atrial Fibrillation
}

\author{
Nikhilesh Bhatt, Thomas Fogarty, Patrick Maguire* \\ Fogarty Institute for Innovation, Mountain View, CA, USA \\ Email: "pmaguire@cyberheartinc.com
}

Received 21 April 2016; accepted 24 May 2016; published 27 May 2016

Copyright (C) 2016 by authors and Scientific Research Publishing Inc.

This work is licensed under the Creative Commons Attribution International License (CC BY). http://creativecommons.org/licenses/by/4.0/

(c) $\underset{\mathrm{EY}}{\mathrm{EY}}$ Open Access

\section{Abstract}

An estimated 4 million Americans currently have atrial fibrillation (AF), the most common form of cardiac arrhythmia. The annual growth rate of $\mathrm{AF}$ is approximately $9.2 \%$ and advanced statistical analyses project that the prevalence of AF will be 12.1 million in the United States by 2030. The current therapeutic options to treat AF put certain patient groups at a high risk for a serious bleeding event, stroke or thromboembolism, myocardial infarction, and death. The most effective treatment for $\mathrm{AF}$ - catheter ablation-has been widely reported to require repeat operations and only leaves $59.4 \%$ of patients AF-free after five years. A majority of the reported complications and increased risks for catheter ablation occur because the procedure is invasive. Thus, there is a need for a novel, non-invasive therapeutic option to treat AF that can potentially expand the treatable patient population, increase patient safety, and improve clinical outcomes. CyberHeart Inc. has been exploring the efficacy and potential of cardiac radiosurgery as an option to treat heart conditions such as AF. With its proprietary CardioPlan ${ }^{\mathrm{TM}}$ software, the CyberHeart System utilizes the Cyberknife stereotactic radiosurgery platform to non-invasively ablate the heart. Early studies have displayed that the CyberHeart System is highly accurate at delivering radiation to cardiac targets and effective at minimizing radiation exposure to healthy tissue that is in close proximity to the targeted anatomic region. The focus of this review is to present the current standard of treatment for AF, identify the specific patient population that is at a high risk for the current treatment modalities, and address the potential for the CyberHeart Stereotactic Radiosurgery System to noninvasively ablate cardiac tissue and return sinus rhythm to patients with AF.

\section{Keywords}

CyberHeart, Cardiac Radiosurgery, Atrial Fibrillation, Non-Invasive, Radiotherapy

\footnotetext{
${ }^{*}$ Corresponding author.
} 


\section{Introduction}

An estimated 4 million Americans currently have atrial fibrillation (AF), the most common form of cardiac arrhythmia [1] [2]. The annual growth rate of AF is approximately $9.2 \%$ and advanced statistical analyses project that the prevalence of AF will be 12.1 million in the United States by 2030 [3]. The hospitalizations with AF as the primary diagnosis exceed 460,000 a year, and AF contributes to more than 80,000 annual deaths [4]. Cost of hospitalization for patients with AF is approximately 3 times more than those without, resulting in an incremental cost for AF that ranges from 6 to 26 billion USD annually [1] [4].

It is reported that $70 \%$ of individuals with $\mathrm{AF}$ have an age between 65 and 85 years [5]. While AF is not thought to be an immediately life-threatening morbidity, it is associated with an elevated risk of death [5]. Symptomatic AF increases risk for stroke or thromboembolism, is associated with increased mortality, and can significantly affect quality of life [1]. Twenty-percent of all strokes are secondary to AF and it has been noted that strokes that result from AF are more disabling and have greater clinical significance than other forms of strokes [6]. From the Framingham cohort, it is found that the total mortality odds ratio is 1.5 and 1.9 for male and female patients with AF, respectively [5] [7]. The length of hospitalizations for patients with AF has been observed to be longer than the recorded times for other patients, and there are significant concerns with the decrease in quality of life that current treatment strategies mandate [5].

The current therapeutic options to treat AF put certain patient groups at a high risk for a serious bleeding event, stroke or thromboembolism, myocardial infarction, and death. This results in a considerable number of patients with AF that are unable to be treated. The most effective treatment for AF is an invasive procedurecatheter ablation - that has been widely reported to require repeat operations and only leaves $59.4 \%$ of patients AF-free after five years [8]. Moreover, certain patient with such as those chronic kidney disease or other co-morbidities is at a high risk for complication for a catheter ablation procedure. The focus of this review is to present the current standard of treatment for AF, identify the specific patient population that is at a high risk for the current treatment modalities, and address the potential for the CyberHeart Stereotactic Radiosurgery System to noninvasively ablate cardiac tissue and the return of sinus rhythm to patients with AF.

\section{Overview of Current Therapies for Atrial Fibrillation}

There are currently two main therapeutic strategies used treat AF: rate control and rhythm control. Rate control strategies typically utilize medicine such as beta-blockers, dihydropyridine CCB, and digoxin [9]. Rhythm control strategies either use antiarrhythmic drugs or invasive catheter ablation procedures. Typical antiarrhythmic drugs include: amiodarone, dronedarone, dofetilide, flecainide, propafenone, and sotalol [9]. For AF patients that are at a high risk for stroke or thromboembolism, antithrombotic drugs such as warfarin, aspirin, prasugrel, and dabigatran are used [9].

\section{Catheter Ablation for the Treatment of Atrial Fibrillation}

Catheter ablation for atrial fibrillation is one of the most complex, technically challenging interventional electrophysiologist procedures [10]. It requires extensive intra-cardiac catheter manipulation and ablation, consistently two or more transseptal punctures, and often-systemic anti-coagulation [10]. As a consequence of the inherent technical demands of this procedure, the risk for AF ablation is often higher than it is for other forms of cardiac arrhythmia [10]. Complications for catheter ablation for AF can result in prolonged hospitalization, long-term disability, and death [10]. Common complications include: cardiac tamponade, pulmonary vein stenosis, esophageal injury, various vascular complications, stroke, phrenic nerve injury, TIA, pericarditis, mitral valve trauma, and others [10] [11].

\subsection{Complications}

In a study conducted by Deshmukh et al., the data for in-hospital complications of catheter ablation for atrial fibrillation were analyzed for 93,801 procedures throughout America from the time period of 2000 to 2010 [12]. The data — which utilized statistics from the Nationwide Inpatient Sample (NIS)—represents approximately 20\% of the community hospitals in the America [12]. The reported incidence of in-hospital deaths from the study was $0.42 \%$. Frequency of procedural complications of any nature was $6.29 \%$, which computes to 5909 complications over the ten-year span of the study [12]. The majority complications for this study occur as result of the proce- 
dure being invasive and can be categorized as: cardiac complications (2.54\%), vascular complications (1.53\%), respiratory complications (1.3\%), and neurological complications (1.02\%) [12]. A year-by-year breakdown of the data displays that the frequency of complications per 100 ablation procedures was 5.33 in 2000 but increased to 7.48 in 2010 [12]. There was not a definitive explanation for the upsurge in complications per 100 patients in 2010. In agreement with previous studies, Deshmukh et al. showed that women notably had an increased complication rate of $7.51 \%$, which was greater the $5.49 \%$ complication rate for men [12]. Another significant trend illustrated is that older patients (age > 80) undergoing catheter ablation have a complication rate of $9.37 \%$, which is greater than the complication rate for younger patients (age < 80) [12]. Other important patterns revealed that complication rates significantly increased for patients with certain comorbidities. Patients with the comorbidities as follows were at a higher risk for complication: history of diabetes (complication rate $=17.83 \%$ ), history of chronic pulmonary disease (complication rate $=23.07 \%$ ), fluid-electrolyte deficiency or renal-failure (complication rate $=23.25 \%$ ), anemia of coagulopathy (complication rate $=14.71 \%$ ) [12] .

The frequency of cardiac tamponade- the most life-threatening complication for patients undergoing catheter AF ablation - in the study by Deshmukh et al. was $1.52 \%$ although, due to limitations of the study, the occurrence of cardiac tamponade could not be tracked beyond hospitalization [12]. Other studies report occurrence of cardiac tamponade at 6\% [12]. In a Worldwide Survey, cardiac tamponade accounted for $25 \%$ of all fatalities for peri-procedural complications [10] [13]. There are three main reasons cardiac tamponade occurs: misdirected transspetal punctures, direct mechanical trauma through the LA appendage, and overheating during radiofrequency energy delivery, which sometimes results in a steam pop [10]. Two Worldwide Surveys of AF Ablation reported 1.2\% and 1.3\% incidence of cardiac tamponade [10] [14] [15]. For catheter ablation procedures that utilized a cryoballoon, the prevalence of cardiac tamponade was 1.5\% [10].

Pulmonary vein stenosis is another complication that may transpire during catheter ablation for AF due to thermal injury to the pulmonary veins and musculature [10] [12]. The Worldwide Survey of AF ablation reported a $0.32 \%$ incidence of acute PV stenosis and 1.3\% incidence of persistent PV stenosis [10]. Additionally, esophageal injury may occur as a consequence of direct thermal injury or ischemic injury from thermal occlusion of end-arterioles [10]. Some forms of esophageal injury such as left atrial-esophageal fistula have a mortality rate of $80 \%$ [10]. The extent of thromboembolism caused by catheter ablation has been reported up to $7 \%$ [10] [16] [17]. The study by Deshmukh et al. reported a combined frequency of TIA and stroke of $1.52 \%$ [12]. Thromboembolisms typically arise within 24 hours of the procedure; however, the high-risk period extends for the first two weeks after the procedure [10] [12]. The published prevalence of vascular complications due to catheter ablation ranges from 0\% to 13\% [10] [12]. Deshmukh et al. discovered a significant increasing trend for vascular complications and reported a vascular complication rate of 3.38\% [12]. Serious vascular complications can lead to substantial morbidity and may require open surgery repair or blood transfusion [10].

\subsection{Recurrences}

Reported recurrences of AF after initial catheter ablation typically range from $20 \%$ to $40 \%$ [10] [18]. A study that followed 1220 patients from 2003 to 2009 tracked the prevalence of repeat procedures of catheter ablation for AF. The study found that 328 (26.9\%) patients required one repeat catheter ablation, and 42 patients (3.4\%) underwent a second repeat ablation [8]. After five-years, 59.4\% of patients were AF-free after one procedure [8]. There were a total of 376 repeat ablation procedures on 328 patients [8]. A later examination of the cohort of patients, inclusive to those that had a second and third catheter ablation procedure, found that restoration of sinus rhythm was only 83.4\% (1018 of 1220 patients) [8]. The study also found that a Left Atrium Diameter indexed to body surface area (LADI) of $24.0 \mathrm{~mm} / \mathrm{m}^{2}$, age of 65 , and a number of ineffective antiarrhythmic drugs are significant clinical predictors of AF recurrence after a catheter ablation procedure [8] [19].

A study by Shah et al. tracked a cohort of 4156 patients using data from the California State Inpatient Database for the time period of 2005 to 2008 [11]. The study, which included rate of repeat procedures for first time patients of catheter ablation for atrial fibrillation, displayed similar results for the necessity of repeat catheter ablation procedures. After one-year, 22\% of patients were re-hospitalized for arrhythmia recurrence or repeat ablation and after two-years 30\% were affected for the same reasons [11]. These reports of frequent recurrence of arrhythmia and requirement for additional ablations are consistent with other studies; namely, a study of randomized controlled trials reported AF recurrence in $13 \%$ to $44 \%$ of AF ablation recipients, and a worldwide survey of centers offering AF ablation reported recurrence in $48 \%$ to $57 \%$ of recipients [11] [15] [20] [21]. For 
patients with risk factors of HTN and hyperlipidemia, the recurrence rate was 75\% [10]. One study that followed patients for up to five years after their first catheter ablation for AF found that only $29 \%$ of patients were AF free after a single ablation procedure; another study that tracked 264 patients reported that the recurrence rate for of AF five years after patients received their first ablation was 25.5\% [11] [22] [23]. The findings that there is a high recurrence of AF after catheter ablation and a consistent necessity for repeat ablation procedures are in complete accord with previous reports [10] [11] [22] [24]-[28].

As aforementioned, catheter ablation is a technically complex procedure. The specific skills required to perform a catheter ablation include transseptal needle puncture and cannulation of the LA, precise control of the catheter for both mapping and ablation, ability to discern the pulmonary ostia, adjustment of the energy used for ablation, use of fluoroscopy, radiographic contrast for imaging, and 3D mapping systems or intra-cardiac echocardiography [10]. The high degree of technical competence and complex nature of performing a catheter ablation for AF may be a reason why there are statistical trends that link the annual operator volume and case volume with the overall frequency of complications per year [12]. More specifically, when there was less than 50 cases a year in a center with less than 25 operators there was a higher chance of an adverse outcome than when there 25 to 50 operators for a center that performed more than 50 cases a year [12].

Another complication trend, which likely occurs as a result of the technical requirements of catheter ablation, is the reported incidence of higher complication rates outside of experienced academic hospitals. The reported rate of procedural complications at experienced academic hospitals has been reported to be between $1 \%$ and $8 \%$, which is less than the procedural complication range reported in non-academic settings (7\% to 10\%) [11] [15] [22] [29]. It is important to realize that the current complications that are prevalent occur due to the invasive nature of the current procedure. However, if a non-invasive, procedure that allowed the operator to easily make highly precise ablations using 3D software (such as CyberHeart) was available, it would be unlikely that trends that result from variation in experience and technical competence occur.

Catheter ablation of AF may not be an appropriate procedure for all ages. It must be acknowledged that there are not currently special catheters that can be used on pediatric patients with AF that possess significantly smaller heart chambers than adults [30]. Accordingly, the degree of difficulty to perform a catheter ablation on a pediatric patient is likely higher and requires the electrophysiologist to be tremendously technically competent. A study conducted by Mills et al. that tracked forty-two pediatric patients from 1996 to 2011 discovered that the rate of recurrence of atrial fibrillation was 39\% [31]. Also, the complication rate for patients that are older than 80 years old is $9.37 \%$ [12]. Elderly patients likely have an increased complication rate due to the normal deterioration of physiological capacity that occurs with age and an increased prevalence of co-morbidities. An entirely non-invasive treatment that can precisely ablate cardiac tissue has the potential to be extremely beneficial and advantageous for the future treatment pediatric and elderly patients.

\section{Risk of Current Therapies for Atrial Fibrillation}

\subsection{Risk of Antiarrhythmic Drugs for AF Treatment}

The use of antiarrhythmic medications to manage AF is therapeutic strategy that is frequently selected [32]. The objective of antiarrhythmic drugs is to reduce the frequency and duration of arrhythmia episodes in addition to reducing mortality and need for hospitalization for AF [32]. Limitations of antiarrhythmic drug therapy include proarrhythmic and non-cardiovascular toxicities, and only having modest antiarrhythmic efficacy [32].

Antiarrhythmic drugs that can lead to a multitude of cardiovascular and non-cardiovascular side effects [32]. Drugs that utilize the potassium channels to slow the heart rate such as sotalol and dofetilide have can have significant effects on potassium currents and prolong the action potential duration and refractory periods [32]. Amiodarone and dronedarone can also affect action potential duration and refractory periods [32].

Amiodarone is the most commonly prescribed antiarrhythmic drug, accounting for $45 \%$ of all U.S. annual drug prescriptions [32]. Sinus bradycardia is the major cardiovascular side effect of amiodarone. Also, QT prolongation that can sometimes be associated with TDP $(<0.5 \%)$ is another possible side effect. Other non cardiovascular side effects include acute hypersensitivity pneumonitis, chronic interstitial infiltrates, hepatitis, thyroid issues, photosensitivity, nausea and others [32].

Approximately 26\% of annual antiarrhythmic drug prescriptions in the US are solatol [32]. Because sotalol is renally cleared, it cannot be prescribed if a patient has creatinine clearance that is less than $30 \mathrm{~mL} / \mathrm{min}$ [32]. A possible risk of sotalol is ventricular proarrhythmia (TDP) due to an increase in potassium channel blocking 
with increased doses. Possible risks of sotalol include brachycardia, torsades de pointes, and bronchospasm [32]. Other drugs such as flecainide and propafenone represent $10 \%$ of the US annual antiarrhythmic drug prescriptions and are typically prescribed to patients with AF that have no structural heart disease. The side effects of both drugs are associated with a significant increase in incidence of atrial flutter [32]-[34]. Also, both flecainide and propafenone are contraindicated for individuals with previous myocardial infarction and reduced left ventricle function because of increased risk of ventricle proarrythmia [32] [35] [36]. For patients taking flecainide, dizziness and visual disturbance occur as side effects in 5\% - 10\% of all patients [32] [35]. Major non-cardiovascular side effects of propafenone include a metallic taste, dizziness, and other visual disturbances [32].

\subsection{Risk of Antithrombotic Drugs for AF Treatment}

Atrial fibrillation increases risk of stroke by a factor of five [37] [38]. To reduce the risk of stroke, antithrombotic drugs are often prescribed to patients with AF; however, antithrombotic drugs also increase risk of bleeding and can lead to death [39]. There are two different categories of oral anticoagulants that are currently used: vitamin K antagonists (VKAs, e.g. warfarin) and non-VKA oral anticoagulants such as direct thrombin inhibitor (dabigatran) or oral factor Xa blockers (rivaroxaban, apixaban, edosaban) [39] [40]. The occurrence of major bleeding events is the primary concern when using antithrombotic drugs [39]. Major bleeding events that can occur are typically defined by the following criteria: fatal bleeding, symptomatic bleeding in a critical area or organ, intraspinal, intraocular, retroperitoneal, intra-articular or pericardial, intramuscular with compartment syndrome, bleeding causing a fall in hemoglobin level of $20 \mathrm{~g} / \mathrm{L}$ or more, or an event that leads to transfusion of two or more units of whole blood or red cells [39] [41].

\subsection{Risk of Catheter Ablation for AF Treatment}

Catheter ablation for AF has the risk of complications that can lead to long-term disability and death [10]. Please see section 3 for additional information on the risk of catheter ablation for AF treatment (chance of complications, recurrence, etc.).

\section{Stereotactic Radiosurgery for the Treatment of AF}

While the current therapeutic options for the treatment of AF do address a significant amount of the population that suffer from the condition, there still remains a notable cohort of individuals with AF and other comorbidities that the current therapies ineffectively treat. The following section identifies a cohort of AF patients with comorbidities that put them at a high risk for the current therapies, the potential role of stereotactic radiosurgery to treat AF, and the CyberHeart Stereotactic Ablative Radiosurgery System.

\subsection{Clinical Population Potentially Addressable with Cardiac Radiosurgery}

A specific clinical population that the current therapeutic strategies do not adequately treat, is patients with AF and chronic kidney disease. Both atrial fibrillation and chronic kidney disease increase the risk of stroke when they are sole morbidities [37]. Atrial fibrillation increases risk of stroke by a factor of 5 and chronic kidney disease has been reported by the U.S. Renal Data System to increase the risk for stroke by a factor of 3.7. End stage-renal disease increases the risk of stroke by a factor of 5.8 [37] [38]. Typically, in order to reduce risk of stroke or systemic thromboembolism anti-coagulants such as warfarin are prescribed [37]. However, multiple studies have indicated that use of warfarin may increase the risk of ischemic stroke for patients undergoing dialysis and the risk of bleeding increases for patients with chronic kidney disease [37] [42]-[44].

A study conducted by Olesen et al. used the Danish National Registry to track 132,372 patients with nonvalvular atrial fibrillation from the time period of 1997-2008 [37]. The study identified 4488 patients of the original cohort that also possessed or developed chronic kidney disease (2.7\%) or end-stage renal disease (0.7\%). Trends for patients with a comorbidity of renal disease showed increased risk factors for stroke or thromboembolism, myocardial infarction, bleeding, and death [37]. The stroke or thromboembolism rate per 100 persons/ year for patients with non-end stage chronic kidney disease (CKD) was 6.44, and for patients that required renal-replacement therapy it was 5.61 [37]. Both stats were greater than the stroke/thromboembolism rate per 100 persons a year for patients without renal disease, which was 3.61. The patient group with no renal disease also had a rate for bleeding events per 100 persons a year that was less than half of those with non-end stage CKD 
and that required renal replacement therapy. Specifically, patients with non-end stage CKD possessed a bleeding rate per 100 persons/year of 8.77 and for patients that required renal-replacement therapy it was 8.89; for the group without renal disease it was only 3.54 [37]. The rates of myocardial infarction per 100 patients/year for patients with non-end stage renal disease and patients that required renal therapies were 5.81 and 5.98, respectively. For patients with no renal disease the rate of myocardial infarction per 100 patients/year was 1.88 . The death rate per 100 patients a year for patients for no renal disease was 11.21 , while the rates were 38.65 and 29.35 for non-end stage CKD and patients that required renal-replacement, respectively [37].

The study by Olesen et al. also showed that patients with or without renal disease that underwent warfarin treatment had a reduced risk for stroke or thromboembolism, but both warfarin and aspirin also increased risk of bleeding [37]. A study by Lip et al. investigated a cohort of 7329 patients with AF that were anti-coagulated orally with adjusted doses of warfarin or two fixed-doses of $36 \mathrm{mg}$ of ximelagatran daily [45]. There were 217 major bleeding events_-defined as fatal bleeding, bleeding that required transfusion of $>2 \mathrm{U}$ of blood, 20 or more g/l decrease in hemoglobin, or bleeding of a critical anatomic site other than the brain-that occurred in groups of the AF patients that possessed: diabetes, LV dysfunction, previous stroke or ischemic attack, and impaired renal function [45]. The hazard ratio (HR) for bleeding was highest for patients with impaired renal function (HR = 2.48); second highest risk for bleeding was for patients with labile INR (HR = 2.14); patients older than 75 had the third highest risk with a HR = 2.10 [45].

There are also variations in the risk for bleeding and stroke or thromboembolism based on the particular renal disease. Oleson et al. showed that patients with chronic glomerulonephritis possessed the highest risk for bleeding out of all the non-end stage renal diseases examined (HR for chronic glomerulonephritis = 2.81) [37]. Diabetic nephropathy also possessed a high hazard ratio $(\mathrm{HR}=1.96)$ for bleeding [37]. Advanced statistical analyses also indicated that patients with hypertensive nephropathy have the highest risk of stroke or thromboembolism (HR = 1.92) [37]. Also, the daily intake of loop diuretics increased risk for stroke/thromboembolism and bleeding. A daily intake of $40-160 \mathrm{mg}$ of furosemide had an increased hazard ratio for stroke $(\mathrm{HR}=1.60)$ and bleeding (HR = 2.41). Greater than $160 \mathrm{mg} /$ daily of furosemide showed similar increased risks for both stroke/ thromboembolism $(\mathrm{HR}=1.51)$ and bleeding $(\mathrm{HR}=2.85)$ [37].

In studies that have analyzed populations with severe renal impairment with AF, it has been found that these patient groups are often diagnosed with ischemic heart disease, valvular heart disease, accelerated vascular calcification, and ventricular hypertrophy [46]-[48]. The important information that this observation provides is that patients with renal-failure and AF often also have abnormal vascular anatomy. A study that analyzed 93,801 catheter ablation procedures for AF identified patients with renal failure are at a higher risk for the procedure citing that the in-hospital rate of complication for renal failure patients was $23.25 \%$ [12]. The increase risk for patients with AF and renal failure undergoing catheter ablation may be a result of the abnormal vascular anatomy that increases the complexity of the operation. Another feasible explanation for the increased risk factor is that catheter ablation procedures can require anti-coagulation up to 3 weeks before a procedure, during the transseptal puncture, and for up to 3-months after the procedure [10]. The necessity to anti-coagulate for catheter ablations may be a reason why there is a higher complication rate for renal-disease patients. As previously mentioned anti-coagulation leads to an increased risk for bleeding. Both possible explanations for increased risk of catheter ablation for AF in patients with renal-disease clearly indicate that a therapeutic option that can perform cardiac ablation non-invasively would likely have significant implications for AF patients.

In sum, patients with AF and the comorbidities of: end-stage renal disease, chronic glomerulonephritis, diabetic nephropathy, and hypertensive nephropathy are at a high risk for the current therapies for the treatment of AF. For these clinical populations with chronic kidney disease and AF, utilizing anti-coagulants to improve their condition puts them at an increased risk of having a serious bleeding event or stroke. Selecting catheter ablation as the treatment strategy can also requires the use of and has the risks anti-coagulation. Additionally, catheter ablation on patients with AF and chronic kidney disease may have an increased level of difficulty because vascular these patients are often observed to have abnormal vascular anatomy. Consequently, another therapeutic option to treat this clinical population with an acceptable level of risk and increased level of efficacy is needed. Stereotactic radiosurgery (SRS) may present a method to non-invasively ablate the heart without the need for anti-coagulation and may be an efficacious way to reduce AF symptoms in patients with AF and chronic kidney disease. Concerns with vascular anatomy being a cause of procedural complication are also avoided with SRS because the procedure is completely non-invasive. Accordingly, the most effective way to return sinus rhythm to patients with AF and chronic kidney disease may be by performing cardiac radiosurgery with a SRS system. 


\subsection{Efficacy and Safety of Stereotactic Radiosurgery}

After it was established that stereotactic radiosurgery can be utilized to improve clinical outcomes for brain tumors treatments in the 1980s, the prevalence of SRS increased and was widely implemented into cancer therapeutic strategies [49]-[51]. Stereotactic radiosurgery and stereotactic body radiation therapy have since been widely observed to be efficacious and safe [49]. When used to treat brain metastases multiple investigations have found that the SRS results in accurate targeting of neoplasms and improved local control rates [49] [52] [53]. The side effects that occur when using SRS are primarily minor, and include headaches, screw site infections, and short exacerbation [49] [54]. The more serious side effects that can occur from SRS include brain edema, radiation necrosis, or the formation of a new cognitive deficiency; however, these side effects are rare and have been observed to happen in less than 5\% of patients [49] [54]. The accuracy of radiation delivered to intracranial sites that are treated with SRS has been reported to be approximately $1 \mathrm{~mm}$ for cranial locations and 1.5 - $2 \mathrm{~mm}$ for anatomical regions besides the cranium [49] [55]-[58]. Such precision is only anticipated to improve as additional technological innovations and advances are made.

\subsection{CyberHeart Stereotactic Ablative Radiotherapy System}

CyberHeart Inc. has been exploring the efficacy and potential of cardiac radiosurgery as an option to treat heart conditions such as AF. The CyberHeart system employs the Cyberknife stereotactic radiosurgery platform-the same technology currently being used deliver precise radiation to brain tumors-to ablate the heart non-invasively. The Cyberknife stereotactic radiosurgery system is highly accurate at delivering radiation to exact anatomic regions while minimizing radiation exposure to healthy tissue that is in close proximity to the targeted anatomic region. The CyberHeart SRS system is capable of guiding the Cyberknife SRS system to ablate the heart and other regions on the body with the same level of accuracy that is used when targeting lesions in the brain.

A recent study established that the Cyberknife SRS system was accurate and efficacious at delivering radiation to a cardiac target. In the study, the pulmonary vein ostia of animal models were irradiated with Cyberknife using $6 \mathrm{MV}$ x-rays to produce a scar for the purpose of blocking aberrant signals that occur in hearts with AF [59]. A radiation dose between 20 - 35 Gy was programmed to be delivered to four different animals [59]. Surgically implanted TLD and MOSET dosimeters measured that the actual radiation delivered to the animals was highly accurate and restricted the intended anatomic locations [59]. The TLD dosimeter measured the dose on the epicardial surface near the right pulmonary vein to average 5\% less than the predicted dose [59]. MOSET sensors measured doses in the coronary sinus to average less than $6 \%$ than the predicted dose [59]. Measurements on the esophagus measured less than $25 \%$ than predicted [59].

Another recent report utilized the CyberHeart system to irradiate the cavo-tricuspid isthmus, AV node, pulmonary vein-left atrial junction, or left atrial appendage in sixteen Hanford-Sinclair mini swine [60]. Prior to radiosurgically ablating the swine, baseline computed tomographic scans and electroanatomic maps were recorded. The animals were examined for 25 - 196 days after the treatment and electrical voltage mapping and transesophageal echocardiography was repeated; after the examination period, animals were sacrificed and pathology specimens were retrieved [60]. Findings indicated that at least $25 \mathrm{~Gy}$ doses were required to cause electrophysiologic effects. Additionally, it was observed that electrophysiologic effects consistently needed 30 - 90 days to take effect. The results showed a significant voltage reduction to less than $0.05 \mathrm{mV}$ to the pulmonary vein-left atrial junction and left appendage after treatment; a result similar to those attained by an invasive catheter ablation procedure. No spontaneous arrhythmias were observed in the swine and pathology specimens showed no proof of radiation damage outside targeted anatomical regions [60]. The specific anatomic sites that were precisely targeted in this study are representative of the CyberHeart system's efficacy to ablate with a high degree of accuracy and potential to noninvasively treat conditions such as AF [60].

In addition to being a completely non-invasive therapeutic option to treat $A F$, it is also important to realize that the CyberHeart system is distinct because it applies a completely anatomic-approach to treat conditions such as $A F$ [60]. A therapeutic option that is non-invasive, painless, and anatomically based such as CyberHeart can potentially have egregious implications for the millions of patients with heart conditions such as atrial fibrillation. The CyberHeart procedure is considered to have an anatomic approach because it allows the physician to select (on a computer software) the precise anatomic locations where the stereotactic surgery system should ablate. Another possible advantage of the CyberHeart system's anatomic approach is the ability to create 
lesions that exactly meet a predetermined shape and size as desired by the physician. This removes concerns about variation of technical ability of physicians and will likely reduce elements of uncertainty that occur during live, invasive procedures. Similarly, it is feasible to predict that the amount of possible complications that will occur from ablation procedures will be reduced because the lesion size will be predetermined. Recall that cardiac tamponade can occur during catheter ablation if the electrophysiologist does not diligently monitor the temperature level in the heart; if the temperature level in the heart rises over a certain value, pops can occur [10] [13]. Furthermore, the CyberHeart therapeutic option may expand the treatable population of patients with heart conditions. The CyberHeart System procedure is completely non-invasive and requires no sedation, which will expand treatment to patients that are at a high risk for invasive procedures or cannot be sedated.

\subsection{Risk of the CyberHeart System for the Treatment of AF}

Although there is documentation that stereotactic radiosurgery has been effective at treating tumors that move during respiration, cardiac motion may be a challenge for treating cardiac conditions such as AF with stereotactic radiosurgery [60] [61]. During respiration, the various phases of contraction and relaxation of the heart must be accounted for when using stereotactic radiosurgery to ablate the organ. Thus, use of an integrated target volume is incorporated in the CyberHeart System, allowing compensation for atrial motion [60]. Other respiration compensations that the system currently incorporates include technologies that help compensate for cardiac motion to prevent radiation exposure to the pulmonary veins. While there may be challenges in the tracking of ventricular motion that may necessitate additional compensations for cardiac motion, the CyberHeart System has been suggested to be accurate by using technologies and planning that account for motion of the target [59]-[61]

Previous uses of external radiation directed at the mediastinum show that pericarditis, wall-motion abnormalities, and late vascular injury may occur [60]. Such occurrences can be attributed to the volume of theradiation dose and volume of the myocardium exposed [60]. It is important, however, to consider that previous radiation studies that reported anatomic injuries did not target highly discrete, limited locations as in the studies that reported use of the CyberHeart System [60]. Studies suggest that a dosage of 25 Gy or larger is required to create an ablation that alters electrophysiological properties [59] [60]. It has been confirmed that radiation scatter and leakage $1 \mathrm{~m}$ from the targeted legion does not exceed $0.05 \%$ of the dosage [60]. For instance, if there was a prescription dose of $40 \mathrm{~Gy}$, the maximum leakage and scatter dose would be $20 \mathrm{mGy}$ [60]. In comparison to the radiation emitted during catheter ablation procedures, the radiation from a CyberHeart System is absolutely acceptable. A study reported the mean fluoroscopy durations for AF procedures was greater than 60 minutes in both left anterior oblique (LAO) and right anterior oblique (RAO) [10]. The mean peak skin doses of radiation were reported to be $1.5 \pm 0.4 \mathrm{~Gy}$ in the LAO and $1.0 \pm 0.5$ in the RAO. The projected length of a CyberHeart System procedure is between 60 to 120 minutes [60]. Both procedures have relatively low radiation exposure.

It has been reported that the time period for CyberHeart System ablation lesion to develop and create desired effects is likely to be 30 days or more [60]. This observation of delayed effects is the reason why the CyberHeart System procedure is a likely a viable option to treat conditions that do not require immediate intervention such as atrial fibrillation. Patients undergoing a catheter ablation procedure typically must wait several months before their procedure, so the delayed effects for 1-month may not be a significant concern [60].

Recent clinical experience in refractory ventricular tachycardia and refractory atrial fibrillation has been favorable. There have been no treatment related adverse events and all patients have experienced a reduction in symptomatic arrhythmia. Additional clinical trials that aim to provide further evidence of the efficacy of CyberHeart Systemare ongoing. [59] (Table 1).

\section{Discussion and Conclusion}

The current therapeutic options to treat AF put certain patients with co-morbitidies at an increased risk and are not adequate for all patient groups. Patients with AF and end stage-renal disease are among those with elevated risks for the current therapies. Specifically, end-stage renal disease has been recorded to increase risk of stroke by a factor of 5.8 and treatment to reduce stroke increases risk of fatal bleeding events. Patients with AF and end stage renal disease are reported to have a death rate of 29.35 per 100 patients, while the death rate for AF patients with no renal disease is 11.21 deaths per 100 patients [39].

Catheter ablation is one of the principle therapeutic options used to treat AF. The procedure requires a considerable level of technical competence to perform and, as with any invasive procedure, possesses the possibility 
Table 1. Comparison of cardiac radiosurgery and catheter ablation for AF treatment.

\begin{tabular}{|c|c|c|c|}
\hline \multirow{2}{*}{$\begin{array}{c}\text { Therapeutic } \\
\text { Method }\end{array}$} & \multicolumn{3}{|c|}{ Characteristics of Treatment Method } \\
\hline & Features & Clinical Benefits & Risks \\
\hline $\begin{array}{c}\text { Cardiac } \\
\text { Radiosurgery }\end{array}$ & $\begin{array}{c}\text { Completely non-invasive } \\
\text { Allows anatomic surgical approach to be } \\
\text { planned with software } \\
\text { Physician not required to be in radiosurgery } \\
\text { suite during procedure } \\
\text { Does not rely on technical competence of } \\
\text { operator } \\
\text { Estimated to take } 30 \text { days for lesion to be } \\
\text { formed after undergooing procedure } \\
\text { Does not require anti-coagulation }\end{array}$ & $\begin{array}{l}\text { Does not require patient to be sedated } \\
\text { Expands treatable patient population } \\
\text { to include patients with comorbidities } \\
\text { at high risk for current therapies } \\
\text { May allow more effective treatments } \\
\text { for elderly and pediatric patients }\end{array}$ & $\begin{array}{c}\text { Procedural complication } \\
\text { may lead to radiation } \\
\text { scatter and leakage that } \\
\text { causes: pericarditis, } \\
\text { wall-motion } \\
\text { abnormalities, or late } \\
\text { vascular injury }\end{array}$ \\
\hline $\begin{array}{l}\text { Catheter } \\
\text { Ablation }\end{array}$ & $\begin{array}{l}\text { Invasive procedure } \\
\text { Depends on technical competence of operator } \\
\text { Leaves } 40.6 \% \text { of patients AF free after } 5 \text { years }\end{array}$ & $\begin{array}{l}\text { Immediately creates lesion } \\
\text { Provides therapy to significant } \\
\text { amount of patients with AF }\end{array}$ & $\begin{array}{l}\text { Procedural complication } \\
\text { may lead to: cardiac } \\
\text { tamponade, pulmonary } \\
\text { vein stenosis, esophageal } \\
\text { injury, stroke, phrenic } \\
\text { nerve injury, death }\end{array}$ \\
\hline
\end{tabular}

for complications. The overall complication frequency of catheter ablation for AF is 6.29\% and possible complications include: cardiac tamponade, pulmonary vein stenosis, esophageal injury, various vascular complications, stroke, phrenic nerve injury, TIA, pericarditis, mitral valve trauma, and others [10] [11]. The rate of recurrence of AF after being treated with catheter ablation is also not ideal. Only 59.4\% of patients are AF free five years after one catheter ablation procedure and even after multiple repeat procedures only 83.4\% of patients have sinus rhythm returned [8]. Furthermore, certain patient groups such as those over 80, and those with renal impairment have higher increased chances of complication and do not make good candidates for an invasive procedure [12]. Respectively, the complication rates for patients over 80 and patients that undergo renal impairment have been reported to be $9.37 \%$ and $23.25 \%$ [12].

The level of difficulty of performing an invasive procedure increases when patients have abnormal vasculature and that can be significant. Thus, it is likely that the primary cause for increases in complication rates of catheter ablation for AF on patients with co-morbidities is due to atypical or diseased vascular anatomy and a non-invasive therapeutic option to treat AF can have significant implications for such patients. The CyberHeart System procedure utilizes a cardiac radiosurgery approach that incorporates the Cyberknife stereotactic radiosurgery system to ablate the heart. In addition to revolutionizing the dynamics of AF treatment by providing a completely non-invasive operation, the CyberHeart System procedure can potentially expand the treatable population, and lead to improved clinical outcomes for AF patients. Another feature that contributes the considerable potential of the CyberHeart System to improve clinical outcomes for patients with AF is that the CyberHeart procedure utilizes a completely anatomic approach to ablate the heart. The anatomic approach of the CyberHeart system allows the physician to select the specific anatomic structures in 3-D space that should be ablated with the use of a computer program. The physician is not required to be in the radiosurgery suite or exposed to any radiation during the ablation procedure. This feature has the potential to remove discrepancies in complication rates that vary with the technical competence of a physician and result in precise ablations that effectively treat AF. Accordingly, the CyberHeart System is a novel platform that has a promising potential to treat patients with AF and other arrhythmias. Ongoing research is hoped to provide necessary evidence required to further validate the CyberHeart Systems efficacy to improve clinical outcomes for patients.

\section{Disclosures}

T. Fogarty and P. Maguire serve on the Board of CyberHeart Inc.

\section{References}

[1] Naderi, S., Wang, Y., Miller, A.L., Rodriguez, F., Chung, M.K., Radford, M.J. and Foody, J.M. (2014) The Impact of Age on the Epidemiology of Atrial Fibrillation Hospitalizations. American Journal of Medicine, 127, 158.e1-158.e7. 
http://dx.doi.org/10.1016/j.amjmed.2013.10.005

[2] Camm, J. (2005) Atrial Fibrillation-An End to the Epidemic? Circulation, 112, iii.

[3] Colilla, S., Crow, A., Petkun, W., Singer, D., Simon, T. and Liu, X.C. (2013) Estimates of Current and Future Incidence and Prevalence of Atrial Fibrillation in the U.S. Adult Population. American Journal of Cardiology, 112, 11421147. http://dx.doi.org/10.1016/j.amjcard.2013.05.063

[4] Kim, M.H., Johnston, S.S., Chu, B.C., Dalal, M.R. and Schulman, K.L. (2011) Estimation of Total Incremental Health Care Costs in Patients with Atrial Fibrillation in the United States. Circulation: Cardiovascular Quality and Outcomes, 4, 313-320. http://dx.doi.org/10.1161/CIRCOUTCOMES.110.958165

[5] Karamichalakis, N., Letsas, K.P., Vlachos, K., Georgopoulos, S., Bakalakos, A., Efremidis, M. and Sideris, A. (2015) Managing Atrial Fibrillation in the Very Elderly Patient: Challenges and Solutions. Vascular Health and Risk Management, 11, 555-562. http://dx.doi.org/10.2147/VHRM.S83664

[6] Kyprianou, K., Pericleous, A., Stavrou, A., Dimitrakaki, I.A., Challoumas, D. and Dimitrakakis, G. (2016) Surgical Perspectives in the Management of Atrial Fibrillation. World Journal of Cardiology, 8, 41-56. http://dx.doi.org/10.4330/wjc.v8.i1.41

[7] Benjamin, E.J., Wolf, P.A., D’Agostino, R.B., Silbershatz, H., Kannel, W.B. and Levy, D. (1998) Impact of Atrial Fibrillation on the Risk of Death: The Framingham Heart Study. Circulation, 98, 946-952. http://dx.doi.org/10.1161/01.CIR.98.10.946

[8] Takigawa, M., Takahashi, A., Kuwahara, T., Okubo, K., Takahashi, Y., Watari, Y., Takagi, K., Fujino, T., Kimura, S., Hikita, H., Tomita, M., Hirao, K. and Isobe, M. (2014) Long-Term Follow-Up after Catheter Ablation of Paroxysmal Atrial Fibrillation: The Incidence of Recurrence and Progression of Atrial Fibrillation. Circulation: Arrhythmia and Electrophysiology, 7, 267-273. http://dx.doi.org/10.1161/CIRCEP.113.000471

[9] Fosbol, E.L., Holmes, D.N., Piccini, J.P., Thomas, L., Reiffel, J.A., Mills, R.M., Kowey, P., Mahaffey, K., Gersh, B.J., Peterson, E.D., the ORBIT-AF Investigators and Patients (2013) Provider Specialty and Atrial Fibrillation Treatment Strategies in United States Community Practice: Findings from the ORBIT-AF Registry. Journal of the American Heart Association, 2, e000110. http://dx.doi.org/10.1161/JAHA.113.000110

[10] Calkins, H., Kuck, K.H., Cappato, R., Brugada, J., Camm, A.J., Chen, S.A., Crijns, H.J., Damiano Jr., R.J., Davies, D.W., DiMarco, J., Edgerton, J., Ellenbogen, K., Ezekowitz, M.D., Haines, D.E., Haissaguerre, M., Hindricks, G., Iesaka, Y., Jackman, W., Jalife, J., Jais, P., Kalman, J., Keane, D., Kim, Y.H., Kirchhof, P., Klein, G., Kottkamp, H., Kumagai, K., Lindsay, B.D., Mansour, M., Marchlinski, F.E., McCarthy, P.M., Mont, J.L., Morady, F., Nademanee, K., Nakagawa, H., Natale, A., Nattel, S., Packer, D.L., Pappone, C., Prystowsky, E., Raviele, A., Reddy, V., Ruskin, J.N., Shemin, R.J., Tsao, H.M. and Wilber, D. (2012) 2012 HRS/EHRA/ECAS Expert Consensus Statement on Catheter and Surgical Ablation of Atrial Fibrillation: Recommendations for Patient Selection, Procedural Techniques, Patient Management and Follow-Up, Definitions, Endpoints, and Research Trial Design. Europace, 14, 528-606. http://dx.doi.org/10.1093/europace/eus027

[11] Shah, R.U., Freeman, J.V., Shilane, D., Wang, P.J., Go, A.S. and Hlatky, M.A. (2012) Procedural Complications, Rehospitalizations, and Repeat Procedures after Catheter Ablation for Atrial Fibrillation. Journal of the American College of Cardiology, 59, 143-149. http://dx.doi.org/10.1016/j.jacc.2011.08.068

[12] Deshmukh, A., Patel, N.J., Pant, S., Shah, N., Chothani, A., Mehta, K., Grover, P., Singh, V., Vallurupalli, S., Savani, G.T., Badheka, A., Tuliani, T., Dabhadkar, K., Dibu, G., Reddy, Y.M., Sewani, A., Kowalski, M., Mitrani, R., Paydak, H. and Viles-Gonzalez, J.F. (2013) In-Hospital Complications Associated with Catheter Ablation of Atrial Fibrillation in the United States between 2000 and 2010: Analysis of 93,801 Procedures. Circulation, 128, 2104-2112. http://dx.doi.org/10.1161/CIRCULATIONAHA.113.003862

[13] Cappato, R., Calkins, H., Chen, S.A., Davies, W., Iesaka, Y., Kalman, J., Kim, Y.H., Klein, G., Natale, A., Packer, D. and Skanes, A. (2009) Prevalence and Causes of Fatal Outcome in Catheter Ablation of Atrial Fibrillation. Journal of the American College of Cardiology, 53, 1798-1803. http://dx.doi.org/10.1016/j.jacc.2009.02.022

[14] Cappato, R., Calkins, H., Chen, S.A., Davies, W., Iesaka, Y., Kalman, J., Kim, Y.H., Klein, G., Natale, A., Packer, D., Skanes, A., Ambrogi, F. and Biganzoli, E. (2010) Updated Worldwide Survey on the Methods, Efficacy, and Safety of Catheter Ablation for Human Atrial Fibrillation. Circulation: Arrhythmia and Electrophysiology, 3, 32-38. http://dx.doi.org/10.1161/CIRCEP.109.859116

[15] Cappato, R., Calkins, H., Chen, S.A., Davies, W., Iesaka, Y., Kalman, J., Kim, Y.H., Klein, G., Packer, D. and Skanes, A. (2005) Worldwide Survey on the Methods, Efficacy, and Safety of Catheter Ablation for Human Atrial Fibrillation. Circulation, 111, 1100-1105. http://dx.doi.org/10.1161/01.CIR.0000157153.30978.67

[16] Chen, S.-A. and Tai, C.-T. (2005) Catheter Ablation of Atrial Fibrillation Originating from the Non-Pulmonary Vein Foci. Journal of Cardiovascular Electrophysiology, 16, 229-232. http://dx.doi.org/10.1046/j.1540-8167.2005.40665.X

[17] Pappone, C., Rosanio, S., Augello, G., Gallus, G., Vicedomini, G., Mazzone, P., Gulletta, S., Gugliotta, F., Pappone, A., Santinelli, V., Tortoriello, V., Sala, S., Zangrillo, A., Crescenzi, G., Benussi, S. and Alfieri, O. (2003) Mortality, 
Morbidity, and Quality of Life after Circumferential Pulmonary Vein Ablation for Atrial Fibrillation: Outcomes from a Controlled Nonrandomized Long-Term Study. Journal of the American College of Cardiology, 42, 185-197. http://dx.doi.org/10.1016/S0735-1097(03)00577-1

[18] Kobza, R., Hindricks, G., Tanner, H., Schirdewahn, P., Dorszewski, A., Piorkowski, C., Gerds-Li, J.H. and Kottkamp, H. (2004) Late Recurrent Arrhythmias after Ablation of Atrial Fibrillation: Incidence, Mechanisms, and Treatment. Heart Rhythm, 1, 676-683. http://dx.doi.org/10.1016/j.hrthm.2004.08.009

[19] Winkle, R.A., Mead, R.H., Engel, G. and Patrawala, R.A. (2011) Long-Term Results of Atrial Fibrillation Ablation: The Importance of All Initial Ablation Failures Undergoing a Repeat Ablation. American Heart Journal, 162, $193-200$. http://dx.doi.org/10.1016/j.ahj.2011.04.013

[20] Wazni, O.M., Marrouche, N.F., Martin, D.O., Verma, A., Bhargava, M., Saliba, W., Bash, D., Schweikert, R., Brachmann, J., Gunther, J., Gutleben, K., Pisano, E., Potenza, D., Fanelli, R., Raviele, A., Themistoclakis, S., Rossillo, A., Bonso, A. and Natale, A. (2005) Radiofrequency Ablation vs Antiarrhythmic Drugs as First-Line Treatment of Symptomatic Atrial Fibrillation: A Randomized Trial. JAMA, 293, 2634-2640. http://dx.doi.org/10.1001/jama.293.21.2634

[21] Stabile, G., Bertaglia, E., Senatore, G., De Simone, A., Zoppo, F., Donnici, G., Turco, P., Pascotto, P., Fazzari, M. and Vitale, D.F. (2006) Catheter Ablation Treatment in Patients with Drug-Refractory Atrial Fibrillation: A Prospective, Multi-Centre, Randomized, Controlled Study (Catheter Ablation for the Cure of Atrial Fibrillation Study). European Heart Journal, 27, 216-221. http://dx.doi.org/10.1093/eurheartj/ehi583

[22] Weerasooriya, R., Khairy, P., Litalien, J., Macle, L., Hocini, M., Sacher, F., Lellouche, N., Knecht, S., Wright, M., Nault, I., Miyazaki, S., Scavee, C., Clementy, J., Haissaguerre, M. and Jais, P. (2011) Catheter Ablation for Atrial Fibrillation: Are Results Maintained at 5 Years of Follow-Up? Journal of the American College of Cardiology, 57, 160166. http://dx.doi.org/10.1016/j.jacc.2010.05.061

[23] Shah, A.N., Mittal, S., Sichrovsky, T.C., Cotiga, D., Arshad, A., Maleki, K., Pierce, W.J. and Steinberg, J.S. (2008) Long-Term Outcome Following Successful Pulmonary Vein Isolation: Pattern and Prediction of Very Late Recurrence. Journal of Cardiovascular Electrophysiology, 19, 661-667. http://dx.doi.org/10.1111/j.1540-8167.2008.01101.x

[24] Ouyang, F., Tilz, R., Chun, J., Schmidt, B., Wissner, E., Zerm, T., Neven, K., Kokturk, B., Konstantinidou, M., Metzner, A., Fuernkranz, A. and Kuck, K.H. (2010) Long-Term Results of Catheter Ablation in Paroxysmal Atrial Fibrillation: Lessons from a 5-Year Follow-Up. Circulation, 122, 2368-2377. http://dx.doi.org/10.1161/CIRCULATIONAHA.110.946806

[25] Sawhney, N., Anousheh, R., Chen, W.C., Narayan, S. and Feld, G.K. (2009) Five-Year Outcomes after Segmental Pulmonary Vein Isolation for Paroxysmal Atrial Fibrillation. The American Journal of Cardiology, 104, 366-372. http://dx.doi.org/10.1016/j.amjcard.2009.03.044

[26] Wokhlu, A., Hodge, D.O., Monahan, K.H., Asirvatham, S.J., Friedman, P.A., Munger, T.M., Cha, Y.M., Shen, W.K., Brady, P.A., Bluhm, C.M., Haroldson, J.M., Hammill, S.C. and Packer, D.L. (2010) Long-Term Outcome of Atrial Fibrillation Ablation: Impact and Predictors of Very Late Recurrence. Journal of Cardiovascular Electrophysiology, 21, 1071-1078. http://dx.doi.org/10.1111/j.1540-8167.2010.01786.x

[27] Medi, C., Sparks, P.B., Morton, J.B., Kistler, P.M., Halloran, K., Rosso, R., Vohra, J.K., Kumar, S. and Kalman, J.M. (2011) Pulmonary Vein Antral Isolation for Paroxysmal Atrial Fibrillation: Results from Long-Term Follow-Up. Journal of Cardiovascular Electrophysiology, 22, 137-141.

[28] Bertaglia, E., Tondo, C., De Simone, A., Zoppo, F., Mantica, M., Turco, P., Iuliano, A., Forleo, G., La Rocca, V. and Stabile, G. (2012) Does Catheter Ablation Cure Atrial Fibrillation? Single-Procedure Outcome of Drug-Refractory Atrial Fibrillation Ablation: A 6-Year Multicentre Experience. Europace, 12, 181-187.

[29] Ellis, E.R., Culler, S.D., Simon, A.W. and Reynolds, M.R. (2009) Trends in Utilization and Complications of Catheter Ablation for Atrial Fibrillation in Medicare Beneficiaries. Heart Rhythm, 6, 1267-1273. http://dx.doi.org/10.1016/j.hrthm.2009.06.009

[30] Kunimoto, S.Y.K., Yamamoto, K., et al. (2013) Potency of Integrating Three-Dimensional Cardiac Magnetic Resonance Imaging into Electroanatomic Mapping to Perform Catheter Ablation in Pediatrics. Journal of Cardiovascular Magnetic Resonance, 15, M9. http://dx.doi.org/10.1186/1532-429X-15-S1-M9

[31] Mills, L.C., Gow, R.M., Myers, K., Kantoch, M.J., Gross, G.J., Fournier, A. and Sanatani, S. (2013) Lone Atrial Fibrillation in the Pediatric Population. Canadian Journal of Cardiology, 29, 1227-1233. http://dx.doi.org/10.1016/j.cjca.2013.06.014

[32] Zimetbaum, P. (2012) Antiarrhythmic Drug Therapy for Atrial Fibrillation. Circulation, 125, 381-389. http://dx.doi.org/10.1161/CIRCULATIONAHA.111.019927

[33] Huang, D.T., Monahan, K.M., Zimetbaum, P., Papageorgiou, P., Epstein, L.M. and Josephson, M.E. (1998) Hybrid Pharmacologic and Ablative Therapy: A Novel and Effective Approach for the Management of Atrial Fibrillation. Journal of Cardiovascular Electrophysiology, 9, 462-469. http://dx.doi.org/10.1111/j.1540-8167.1998.tb01837.x 
[34] Nabar, A., Rodriguez, L.M., Timmermans, C., van Mechelen, R. and Wellens, H.J. (2001) Class IC Antiarrhythmic Drug Induced Atrial Flutter: Electrocardiographic and Electrophysiological Findings and Their Importance for Long Term Outcome after Right Atrial Isthmus Ablation. Heart, 85, 424-429. http://dx.doi.org/10.1136/heart.85.4.424

[35] Gentzkow, G.D. and Sullivan, J.Y. (1984) Extracardiac Adverse Effects of Flecainide. The American Journal of Cardiology, 53, B101- B105. http://dx.doi.org/10.1016/0002-9149(84)90511-3

[36] Echt, D.S., Liebson, P.R., Mitchell, L.B., Peters, R.W., Obias-Manno, D., Barker, A.H., Arensberg, D., Baker, A., Friedman, L., Greene, H.L., et al. (1991) Mortality and Morbidity in Patients Receiving Encainide, Flecainide, or Placebo-The Cardiac Arrhythmia Suppression Trial. The New England Journal of Medicine, 324, 781-788. http://dx.doi.org/10.1056/NEJM199103213241201

[37] Olesen, J.B., Lip, G.Y., Kamper, A.L., Hommel, K., Kober, L., Lane, D.A., Lindhardsen, J., Gislason, G.H. and TorpPedersen, C. (2012) Stroke and Bleeding in Atrial Fibrillation with Chronic Kidney Disease. The New England Journal of Medicine, 367, 625-635. http://dx.doi.org/10.1056/NEJMoa1105594

[38] (1994) Risk Factors for Stroke and Efficacy of Antithrombotic Therapy in Atrial Fibrillation: Analysis of Pooled Data from Five Randomized Controlled Trials. Archives of Internal Medicine, 154, 1449-1457.

[39] Dzeshka, M.S. and Lip, G.Y.H. (2014) Stroke and Bleeding Risk Assessment: Where Are We Now? Journal of Atrial Fibrilation, 6, 49-57.

[40] Camm, A.J., Lip, G.Y., De Caterina, R., Savelieva, I., Atar, D., Hohnloser, S.H., Hindricks, G., Kirchhof, P., et al. (2012) 2012 Focused Update of the ESC Guidelines for the Management of Atrial Fibrillation: An Update of the 2010 ESC Guidelines for the Management of Atrial Fibrillation Developed with the Special Contribution of the European Heart Rhythm Association. Europace, 14, 1385-1413. http://dx.doi.org/10.1093/europace/eus305

[41] Schulman, S., Kearon, C. and The Subcommittee on Control of Anticoagulation of the Scientific and Standardization Committee of the International Society on Thrombosis and Haemostasis (2005) Definition of Major Bleeding in Clinical Investigations of Antihemostatic Medicinal Products in Non-Surgical Patients. Journal of Thrombosis and Haemostasis, 3, 692-694. http://dx.doi.org/10.1111/j.1538-7836.2005.01204.X

[42] Wizemann, V., Tong, L., Satayathum, S., Disney, A., Akiba, T., Fissell, R.B., Kerr, P.G., Young, E.W. and Robinson, B.M. (2010) Atrial Fibrillation in Hemodialysis Patients: Clinical Features and Associations with Anticoagulant Therapy. Kidney International, 77, 1098-1106. http://dx.doi.org/10.1038/ki.2009.477

[43] Chan, K.E., Lazarus, J.M., Thadhani, R. and Hakim, R.M. (2009) Warfarin Use Associates with Increased Risk for Stroke in Hemodialysis Patients with Atrial Fibrillation. Journal of the American Society of Nephrology, 20, 22232233.

[44] Yang, F., Chou, D., Schweitzer, P. and Hanon, S. (2010) Warfarin in Haemodialysis Patients with Atrial Fibrillation: What Benefit? Europace, 12, 1666-1672. http://dx.doi.org/10.1093/europace/euq387

[45] Lip, G.Y., Frison, L., Halperin, J.L. and Lane, D.A. (2011) Comparative Validation of a Novel Risk Score for Predicting Bleeding Risk in Anticoagulated Patients With Atrial Fibrillation: The HAS-BLED (Hypertension, Abnormal Renal/Liver Function, Stroke, Bleeding History or Predisposition, Labile INR, Elderly, Drugs/Alcohol Concomitantly) Score. Journal of the American College of Cardiology, 57, 173-180. http://dx.doi.org/10.1016/j.jacc.2010.09.024

[46] Marinigh, R., Lane, D.A. and Lip, G.Y. (2011) Severe Renal Impairment and Stroke Prevention in Atrial Fibrillation: Implications for Thromboprophylaxis and Bleeding Risk. Journal of the American College of Cardiology, 57, 13391348. http://dx.doi.org/10.1016/j.jacc.2010.12.013

[47] Abbott, K.C., Trespalacios, F.C., Taylor, A.J. and Agodoa, L.Y. (2003) Atrial Fibrillation in Chronic Dialysis Patients in the United States: Risk Factors for Hospitalization and Mortality. BMC Nephrology, 4, 1. http://dx.doi.org/10.1186/1471-2369-4-1

[48] Vazquez, E., Sanchez-Perales, C., Garcia-Garcia, F., Castellano, P., Garcia-Cortes, M.J., Liebana, A. and Lozano, C. (2009) Atrial Fibrillation in Incident Dialysis Patients. Kidney International, 76, 324-330. http://dx.doi.org/10.1038/ki.2009.185

[49] Solberg, T.D. and Medin, P.M. (2010) Quality and Safety in Stereotactic Radiosurgery and Stereotactic Body Radiation Therapy: Can More Be Done? Journal of Radiosurgery and SBRT, 1, 13-19.

[50] Betti, O.O. and Derechinsky, Y. (1984) Hyperselective Encephalic Irradiation with Linear Accelerator. In: Gybels, J., Hitchcock, E.R., Ostertag, C., Rossi, G.F., Siegfried, J. and Szikla, G., Eds., Advances in Stereotactic and Functional Neurosurgery 6, Springer, Vienna, 385-390.

[51] Colombo, F., Benedetti, A., Pozza, F., Avanzo, R.C., Marchetti, C., Chierego, G. and Zanardo, A. (1985) External Stereotactic Irradiation by Linear Accelerator. Neurosurgery, 16, 154-160. http://dx.doi.org/10.1227/00006123-198502000-00005

[52] Kondziolka, D., Patel, A., Lunsford, L.D., Kassam, A. and Flickinger, J.C. (1999) Stereotactic Radiosurgery plus Whole Brain Radiotherapy versus Radiotherapy Alone for Patients with Multiple Brain Metastases. International 
Journal of Radiation Oncology*Biology*Physics, 45, 427-434. http://dx.doi.org/10.1016/S0360-3016(99)00198-4

[53] Linskey, M.E., Andrews, D.W., Asher, A.L., Burri, S.H., Kondziolka, D., Robinson, P.D., Ammirati, M., Cobbs, C.S., Gaspar, L.E., Loeffler, J.S., McDermott, M., Mehta, M.P., Mikkelsen, T., Olson, J.J., Paleologos, N.A., Patchell, R.A., Ryken, T.C. and Kalkanis, S.N. (2010) The Role of Stereotactic Radiosurgery in the Management of Patients with Newly Diagnosed Brain Metastases: A Systematic Review and Evidence-Based Clinical Practice Guideline. Journal of Neuro-Oncology, 96, 45-68. http://dx.doi.org/10.1007/s11060-009-0073-4

[54] Suh, J. (2010) Stereotactic Radiosurgery for the Management of Brain Metastases. The New England Journal of Medicine, 362, 1119-1127. http://dx.doi.org/10.1056/NEJMct0806951

[55] Chang, S.D., Main, W., Martin, D.P., Gibbs, I.C. and Heilbrun, M.P. (2003) An Analysis of the Accuracy of the CyberKnife: A Robotic Frameless Stereotactic Radiosurgical System. Neurosurgery, 52, 140-146; Discussion 146-147.

[56] Solberg, T.D., Medin, P.M., Mullins, J. and Li, S. (2008) Quality Assurance of Immobilization and Target Localization Systems for Frameless Stereotactic Cranial and Extracranial Hypofractionated Radiotherapy. International Journal of Radiation Oncology*Biology*Physics, 71, S131-S135. http://dx.doi.org/10.1016/j.ijrobp.2007.05.097

[57] Jin, J.Y., Yin, F.F., Tenn, S.E., Medin, P.M. and Solberg, T.D. (2008) Use of the BrainLAB ExacTrac X-Ray 6D System in Image-Guided Radiotherapy. Medical Dosimetry, 33, 124-134. http://dx.doi.org/10.1016/j.meddos.2008.02.005

[58] Furweger, C., Drexler, C., Kufeld, M., Muacevic, A., Wowra, B. and Schlaefer, A. (2010) Patient Motion and Targeting Accuracy in Robotic Spinal Radiosurgery: 260 Single-Fraction Fiducial-Free Cases. International Journal of Radiation Oncology*Biology*Physics, 78, 937-945. http://dx.doi.org/10.1016/j.ijrobp.2009.11.030

[59] Gardner, E.A., Sumanaweera, T.S., Blanck, O., Iwamura, A.K., Steel, J.P., Dieterich, S. and Maguire, P. (2012) In Vivo Dose Measurement Using TLDs and MOSFET Dosimeters for Cardiac Radiosurgery. Journal of Applied Clinical Medical Physics, 13, 3745.

[60] Sharma, A., Wong, D., Weidlich, G., Fogarty, T., Jack, A., Sumanaweera, T. and Maguire, P. (2010) Noninvasive Stereotactic Radiosurgery (CyberHeart) for Creation of Ablation Lesions in the Atrium. Heart Rhythm, 7, 802-810. http://dx.doi.org/10.1016/j.hrthm.2010.02.010

[61] Klemm, H.U., Franzen, O., Ventura, R. and Willems, S. (2007) Catheter Based Simultaneous Mapping of Cardiac Activation and Motion: A Review. Indian Pacing and Electrophysiology Journal, 7, 148-159. 